\title{
The role of Angiology and Vascular Surgery in the COVID-19 pandemic
}

\section{A atuação da Angiologia e da Cirurgia Vascular na pandemia de COVID-19}

Bernardo Cunha Senra Barros $1,2,3$ (iD; Aline Barbosa Maia ${ }^{2}$; Marcos Arêas Marques ${ }^{2,4}$; Paulo Roberto Prette-Junior²; Stenio Karlos Alvim Fiorelli2; Fernanda De Castro Cerqueira, ACBC-RJ33.

\begin{abstract}
A B S T R A C T
The New Coronavirus Epidemic (2019-nCoV), discovered in the city of Wuhan, China, in December 2019, presents mainly with pulmonary pneumonia that is preceded by fever, cough and myalgia. However, as the disease spread globally and the number of hospitalizations increased exponentially, it was noted that most serious patients hospitalized by COVID-19 have laboratory changes worthy of attention, such as lymphopenia, neutrophilia, increased time of prothrombin and increased levels of D-dimer. Due to these changes proving to be crucial for the mortality and morbidity rates in this subset of infected people, several studies focusing on the pathophysiology, mainly hematological, of the disease appear every day. Deepening these studies, several published works have shown SarsCoV-2 infection to the installation of a prothrombotic state in hospitalized patients, which leads to the potential occurrence of thrombotic or arterial events in this cohort. Thus, in order to understand how the departments of Angiology and Vascular Surgery are acting in the context of the COVID-19 pandemic, this work aims to gather studies that reveal from protocols applied in vascular services in the current situation, until to the role of vascular surgeons and angiologists in the clinical and surgical management of patients infected or not, as a way of helping and clarifying this specialty during the context of a pandemic due to the new coranavirus. For the selection of works, the following search criteria were used: "Coronavirus and venous thrombosis", "Coronavirus and thrombosis", "COVID-19 and venous thrombosis" and "COVID-19 Coronavirus and thrombosis".
\end{abstract}

Keywords: Coronavirus Infections. Blood Coagulation. Thrombosis. Pulmonary Embolism. Coronavirus.

\section{INTRODUCTION}

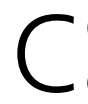
OVID-19 is an infectious disease caused by a newly discovered coronavirus subtype (Sars-CoV-2) in the city of Wuhan, China, in December 2019. It has already infected more than two million people around the world until the date of preparation of this manuscript, according to data from the World Health Organization'.

It is a disease with a broad clinical spectrum, and may present asymptomatically or as a flu syndrome, with symptoms such as fever, cough and myalgia, in addition to evolving in some cases to severe pneumonia or severe acute respiratory syndrome (SARS) ${ }^{2}$.

Laboratory changes such as lymphopenia, neutrophilia, increased prothrombin time and elevated D-dimer (DD) are found in patients who have an unfavorable course of the disease ${ }^{3}$. In addition, the study by Zhou et al. ${ }^{4}$ with 191 patients from Jinyintan Hospitals and the Wuhan Pulmonary Hospital proposed that advanced age, higher SOFA score and DD greater than $1 \mu \mathrm{g} / \mathrm{mL}$ at admission were associated with a higher risk of death; approximately $90 \%$ of hospitalized patients with pneumonia also displayed hypercoagulability ${ }^{4}$. The same study also stated that the possible contributory mechanisms for the systemic changes found in such patients include: (1) the formation of pro-inflammatory cytokines, which are mediators of atherosclerosis, contributing directly to the rupture of the atherosclerotic plaque by local inflammation, (2) induction of procoagulant factors, and (3) hemodynamic changes that predispose to ischemia and thrombosis.

Thus, hospitalized and critically ill patients have hematological changes that can be proven, for example, by high DD rates. However, the relationship

1 - State University of Rio de Janeiro, Department of Vascular Surgery - Rio de Janeiro - RJ - Brazil 2 - Federal University of the State of Rio de Janeiro, Department of Vascular Surgery - Rio de Janeiro - RJ - Brazil 3 - University of the State of Rio de Janeiro, Laboratory of Physiology and Microcirculation (BIOVASC) - Rio de Janeiro - RJ - Brazil 4 - Rio de Janeiro State University, Pedro Ernesto University Hospital (HUPE), Angiology Service - Rio de Janeiro $-R J$ - Brazil 
between the Sars-Cov-2 virus and coagulation mechanisms is still poorly explored in recent studies. Han et al. ${ }^{5}$ demonstrated that antithrombin, a natural anticoagulant, values in patients with COVID-19 were lower, while DD and other fibrin degradation product (FDP) were higher when compared with the control, healthy population. Blood coagulation in these patients thus appears to be altered, and FDP, including DD, can be predictive of disease progression. Therefore, the routine monitoring of such markers is advisable in patients with COVID-19, this result being similar to those found by Tang, Li, Wang and Sun ${ }^{6}$. As a result of the presumed prothrombotic status of many patients, there was a drastic increase in the demand for laboratory tests to monitor coagulation parameters, vascular ultrasound tests and other imaging methods in patients positive for COVID-19. This study aims to gather information about the routine work of vascular specialists and reference centers in the context of the COVID-19 pandemic. We used the following search criteria: "Coronavirus and venous thrombosis", "Coronavirus and thrombosis", "COVID-19 and venous thrombosis" and "COVID-19 Coronavirus and thrombosis".

\section{FINDINGS}

\section{Diagnosis and treatment of venous thromboembolism (VTE) during the COVID-19 pandemic}

In line with recent studies that revealed the high risk of patients hospitalized with COVID-19 to develop thromboembolic events, the Departments of Angiology and Vascular Surgery of hospitals around the world were challenged due to the massive requirement for computed tomography (CT) scans and echo-colorDoppler (ECD) for the diagnosis of pulmonary embolism (PE) and deep vein thrombosis (DVT), respectively, in these patients. Review the evidence for the diagnosis and treatment of VTE has become essential, as well as adapting clinical algorithms and flowcharts for use during the current world scenario. In addition, therapy recommendations for these patients is another critical point that must be approached with care, as the use of anticoagulants in patients who require invasive ventilation creates a potential bleeding risk. Therefore, the risk-benefit must always be reassessed during the clinical course.

To act in this scenario, the University of Michigan Health System, for example, published a complete study ${ }^{7}$ on the creation of a temporary committee of specialists in VTE, vascular surgeons and clinics, to review management protocols already established in the institution. This committee would consult with other specialists in areas such as pulmonology, hematology and medical clinic, among others, so that a new action plan could be developed and executed to better serve patients during the pandemic. For the decision on the use of VTE prophylaxis, patients underwent assessment by the Caprini score $^{8}$ at the time of hospital admission. Although there are not enough data to determine the incidence of VTE in patients positive for COVID-19, there is already evidence showing a reduction in mortality in patients with highly elevated DD ( $>3 x$ the upper normal limit) receiving thromboprophylaxis with non-fractionated (NFH) or low molecular weight (LMWH) heparin?. In addition, VTE and primary pulmonary thrombus may be underlying etiologies potentially responsible for the mortality of critically ill patients due to COVID-19. In view of these findings and the fact that less critical patients have a low risk of bleeding if they use thromboprophylaxis, the study committee recommends routine thromboprophylaxis for all patients hospitalized due to COVID-19, regardless of risk stratification.

As for the suspected diagnosis of PE, Obi et al analyzed two variables: the modified Wells score and whether the patient was intubated or not? ${ }^{7}$.

Table 1. Suggested approach when suspecting pulmonary embolism and Wells score $<4$.

Orotracheal intubation

No

Yes

Orotracheal intubation

Prophylaxis

Empiric anticoagulation low dose

High risk of bleeding
Prophylaxis

Prophylaxis 
Table 2. Suggested approach when suspecting pulmonary embolism and Wells score $>4^{7}$.

\begin{tabular}{|c|c|c|c|}
\hline $\begin{array}{l}\text { Low risk of } \\
\text { bleeding }\end{array}$ & \multicolumn{3}{|c|}{ Anticoagulation } \\
\hline \multirow[t]{3}{*}{$\begin{array}{l}\text { High risk of } \\
\text { bleeding }\end{array}$} & $C T^{*}$ & Negative & $\begin{array}{c}\text { Empiric } \\
\text { anticoagulation - } \\
\text { low dose }\end{array}$ \\
\hline & Positive & OTI† & Anticoagulation \\
\hline & & $\begin{array}{l}\text { No } \\
\text { OTI }\end{array}$ & Anticoagulation \\
\hline
\end{tabular}

* CT: Computed tomography; +OTI: orotracheal intubation.

The study did not recommend the use of ECD to exclude the diagnosis of PE due to its low accuracy. Negative DD associated with a low modified Wells score were considered sufficient to rule out the diagnosis. However, in the context of Sars-Cov-2 infection, which leads to an increase in DD values, its usefulness becomes compromised, which does not mean that its values should be ignored.

In intubated patients without SARS, there is a lack of data on the risk-benefit ratio of empirical anticoagulation strategies. The use of anticoagulation in doses lower than the full dose but higher than the prophylactic dose in patients with SARS was considered based on a previous experience of the team during the $2009 \mathrm{H} 1 \mathrm{~N} 1$ pandemic, but this strategy has not been tested in the current situation?

For suspected DVT, the Michigan Medicine team recommends the use of the Wells score, even though they recognize its limitation in the hospital environment. Its performance during the pandemic pneumonia scenario has not yet been proven on a large scale.

The use of DD dosage should not be the main criterion for performing imaging tests, as it is expected to be high in COVID-19, that is, other clinical manifestations of DVT should be evaluated before requesting such tests for its final diagnosis.

Finally, the Michigan Medicine team touch on long-term anticoagulant therapy. In patients treated with anticoagulants and who are unable to undergo imaging tests for diagnostic purposes during the COVID-19 outbreak, they recommend treatment with direct oral anticoagulants or vitamin $\mathrm{K}$ antagonists for one to two months after hospital discharge, until an image examination can be done. For patients considered to be at moderate to high risk for $\mathrm{PE}$, a CT scan should be requested within one month once they are considered negative for COVID-19. For patients considered to be at high risk for DVT, a lower limb ECD should be requested again once they are considered negative for COVID-19. All patients should be followed and provided recommendations for long-term duration of therapy?.

For Casini et al. ${ }^{9}$, all patients hospitalized with COVID-19 should receive pharmacoprophylaxis, unless contraindicated. Thus, they recommend the use of LMWH in patients with creatinine clearance $>30 \mathrm{~mL} / \mathrm{min}$ and $\mathrm{NFH}$ in patients with clearance $<30 \mathrm{~mL} / \mathrm{min}$, increasing the dose in both cases in patients over $100 \mathrm{~kg}$. They also guide the regular monitoring (daily or two to three times a week) of all patients with prothrombin time, DD, fibrinogen, platelet count, $L D H$, creatinine and ALT, in addition to antithrombin if the patient has DIC, coagulopathy induced by sepsis or heparin resistance, and anti-Xa activity in selected cases. One should consider the use of therapeutic doses of NFH or LMWH in ICU patients with increased DD, signs of severe inflammation, or signs of hepatic or renal dysfunction.

Table 3. Suggested approach supported by the Wells score when suspecting deep venous thrombosis?

\begin{tabular}{|c|c|c|c|}
\hline Wells score $<2$ & \multicolumn{2}{|c|}{ Not intubated } & Prophylaxis \\
\hline & Intubated and criti & - low risk of bleeding & Low dose empiric anticoagulation \\
\hline \multirow[t]{4}{*}{ Wells score $>2$} & VTE-BLEED* $<2$ & Not intubated & Anticoagulation \\
\hline & & Intubated and critical & Low dose empiric anticoagulation \\
\hline & VTE-BLEED* $>2+$ & Negative & Tromboprofilaxia \\
\hline & $\begin{array}{l}\text { With CT or other } \\
\text { image method }\end{array}$ & Positive & Anticoagulation or vena cava filter \\
\hline
\end{tabular}

\footnotetext{
* Risk score for major bleeding in patients with venous thromboembolism.
} 
In Italy, Marietta et al. ${ }^{10}$ published a study in which they guide some care regarding hemostasis when approaching the patient with COVID-19.

- In the management of confirmed patients, laboratory monitoring should always include hemostatic function, platelet count and DVT testing, when possible.

- The use of prophylactic doses of fondaparinux, LMWH or NFH is strongly advised in all hospitalized patients, or mechanical prophylaxis (limb compression) if not possible.

- Pharmacoprophylaxis should be administered during the entire hospitalization and up to seven to 14 days after discharge or in the pre-hospital phase if the case of persisting or pre-existing VTE risks factors.

- Intermediate doses of LMWH can be considered in patients with multiple risk factors for VTE.

- Therapeutic doses of LMWH or NFH are recommended only in patients diagnosed with VTE or as a bridging strategy in patients who already use oral anticoagulants, since the use of oral anticoagulants can represent significant interference in the antiretroviral treatment of patients with COVID-19.

- In patients using full doses, one should consider the dosage of anti-Xa factor or oral anticoagulants in plasma.

We should note that several studies have shown that the patient with COVID-19 has a state of hypercoagulability 2,5,6,11,12 and that, therefore, the pulmonary microembolism index is probably higher than reported. Hence, it is also possible that PE is already present in many patients before admission, justifying the decreased response to thromboprophylaxis in the hospital environment.

Testa et al. ${ }^{13}$, from Cremona, Italy, shared the protocol used in their Hospital for the transition from oral to parenteral anticoagulant in patients with COVID-19.

Table 4. Transiction protocol from oral to parenteral anticoagulation in patients COVID-197.

$\begin{array}{lrl}\text { Medication } & \text { FA não valvar } & \begin{array}{c}\text { Cardiologic valves } \\ \text { Mechanics or } \\ \text { Recent biologic }\end{array}\end{array}$

Antagonists of vitamine $\mathrm{K}$ Suspend the $A V^{*}$ * when INR $<2,0$

HBPM or HNF if ClCreat $<15 \mathrm{~mL} / \mathrm{min}$ in therapeutic dose

HBPM or HNF if CICreat $<15 \mathrm{~mL} / \mathrm{min}$ in therapeutic dose

Suspend the AVK when INR $<2,0$

Do not suspend AVK + daily control of INR

If not possible maintain the AVK, use HBPM 100 UI/kg or HNF with keep anti-Xa control within the superior limit

$\begin{array}{lll}\text { Anticoagulants } & \text { Suspend } & \text { Suspend } \\ \text { direct oral } & \text { the ACODs } & \text { the ACODst }\end{array}$

After $12-24 h$ Start the HBPM or HNF if ClCreat $<15 \mathrm{~mL} / \mathrm{min}$ in therapeutic dose
After 12-24h Start the HBPM or HNF if ClCreat $<15 \mathrm{~mL} / \mathrm{min}$ in therapeutic dose 


\section{The role of Vascular Surgery}

Due to the sudden increase in hospitalizations to meet the needs of COVID-19 critically ill patients, several hospital units found themselves in need of restructuring their services.

The Vascular Surgery Unit of a tertiary hospital in Singapore ${ }^{14}$, for example, has reallocated $25 \%$ of its staff to act on the front line of pandemic care. This rearrangement was necessary both because of the increased demand for health professionals, regardless of the specialty, which the moment requires, and because of the need to care for patients who need some surgical intervention, but who also have symptoms of SarsCoV-2 infection. In addition, to keep the emergency and elective Vascular Surgery Services functioning, two to three surgeons stayed at the Tan Tock Seng Hospital, while the rest of the team was at the National Center for Infectious Diseases, attending to the cases of COVID-19. Among the measures adopted, we highlight:

- Suspension of non-essential meetings, maintaining the use of digital platforms.

- Essential meetings with a maximum of ten people, respecting the use of masks and a distance of one meter between participants. In addition, carrying out daily visits with a small number of people, the patients with suspected or confirmed COVID-19 being seen by only one person in charge of the team.

- Outpatient vascular surgery operation only for diabetic foot care, as well as reviewing the need for care every two to three weeks before the scheduled date of those who needed surgery or symptomatic patients.

- Postponement of consultations for chronic patients, ensuring medications supply and offering home delivery service.

- Maintenance only of the elective surgeries that would have an immediate impact on patients' quality of life and on mortality or would reduce the risk of amputation.

- Screening for symptoms suggestive of COVID-19 infection in patients undergoing surgery at the time of admission and referral of positive cases to the emergency room.

- In the operative act, other measures such as the permanence of the team outside the room during anesthetic induction and recovery.

- The use of personal protective equipment (PPE), consisting of N95 mask or air purifying respirator, goggles, sterile waterproof suits and double layer of sterile gloves by the surgical team, which must be composed of the as few people as possible. The study indicates that the air-purifying respirator is ideal for prolonged surgeries (more than 3 hours), as it is more comfortable for the surgeon. A relevant point is that vascular surgeons should be aware of the complexity of using magnifying surgical loupes associated with the use of PPE when operating suspected or positive COVID-19 patients, especially when performing their removal and cleaning.

The Vascular and Endovascular Surgery Section of the Heart Institute of Minneapolis, following the recommendations of the American College of Surgeons ${ }^{15}$ for COVID in elective procedures, instituted drastic changes in its clinical practice. The unit chose to postpone all elective procedures but those considered essential, whose absence could in some way threaten patients' life or limbs integrity. To ensure that postponed procedures are not forgotten, a database was developed so that rescheduling can be carried out later. Most outpatient visits were converted to telephone visits, limiting the review of medical records and imaging exam to protect the clinical staff ${ }^{16}$.

In Italy, the Department of Vascular and Endovascular Surgery at the Hospital San Giovanni di Dio in Florence adopted a reorganization protocol, also creating a Surgical Vascular Sector for urgent cases of COVID-19. The main measures were:

- Suspension of all varicose vein surgeries and office and emergency activities.

- Centralization of aneurysm and symptomatic carotid artery procedures, as well as vascular surgery to correct critical limb ischemia (CLI) and arteriovenous fistula for hemodialysis.

- Prioritization of endovascular treatment over bypasses, in order to eliminate the need for an anesthesiologist in the operating room.

- Asymptomatic carotid lesions referred to a private hospital that was available to perform them during this period.

- Urgent cases of hemodynamically stable, 
confirmed or suspected, COVID-19 patients from peripheral hospitals centralized to a COVID-19-dedicated Surgical Sector created with a wing isolated from the other operational sectors, converted into an operating room and ICU beds, with an emergency transfer area with an exclusive elevator.

- Standardization of the professionals' equipping sequence, in which surgeons access a room, where they dress with PPE, including N95 mask, goggles, face shield, sterile waterproof suits, foot protectors and two pairs of surgical gloves. Then, the surgeons go to the operating room of the COVID-19 area, where an alcoholic solution is applied to the second pair of gloves, a sterile gown and an extra pair of sterile surgical gloves are placed. After the surgery is completed, surgeons proceed to a room located in front of the operating room, as on a production line, to remove the PPE under the supervision of a trained operator.

- Postoperative evaluation made by two doctors, with only one contacting the patient using the PPE, and the other waiting outside the room, considered "uncontaminated", in order to reduce the risk of cross contamination in the health team.

- Some surgeons stayed in their homes for a few days alternately, in order to preserve a set of uninfected professionals who could be used at any time in case of need or to replace infected colleagues of any specialty.

In this established care algorithm, the clear separation between COVID and non-COVID hospitals, with at least one mandatory test for all admitted patients, was the first step in organizing and restructuring care during the outbreak ${ }^{17}$.

\section{DISCUSSION}

A better understanding of the mechanisms of pathological changes in critically ill patients hospitalized by COVID-19 has been increasingly the focus of studies that aim to define more assertive therapies and better clinical monitoring during the hospitalization of such patients. Several clinical studies have revealed that the "inflammatory cytokines storm"18-20 is an important mechanism implicated in the worsening and mortality of patients with COVID-19. One study revealed that IL-6 levels in critically ill patients were significantly higher than in mild cases, evidencing the contribution of this cytokine to many pathophysiological effects in critically ill patients such as vascular leakage, complement activation and coagulation cascade and induction, in very rare cases, of disseminated intravascular coagulation (DIC). The same study showed that the use of LMWH improves the coagulation dysfunction of patients with COVID-19, as it has anti-inflammatory effects, that is, its use can contribute to prevent or even reverse the cytokine storm, thus improving the condition of the patients, and corroborating its potential therapeutic use ${ }^{21}$. A study conducted at the Weill Cornell Medicine in New York followed this intention to deepen the pathophysiological knowledge on the disease using pulmonary and skin biopsy and autopsy samples and from five individuals with severe COVID-19. They found that at least some patients infected with SARS-CoV-2 who became seriously ill suffered a generalized thrombotic microvascular lesion that appears to be mediated by intense complement activation. Although their five evaluated cases rendered generalization impossible, there is a suspicion that a subset of critically ill patients with COVID-19 may, together with their pro-coagulant state, have associated microvascular lesions that are relevant to the unfavorable outcome that most of these patients have ${ }^{22}$. Thus, the complement system can play a critical role in the pathogenicity of the disease and further studies in this area need to be developed.

Despite advances in publications around the relationship between COVID-19 and the prothrombotic condition that patients have been presenting, so far there are no complete studies associating VTE with COVID-19 infection. However, Cui et al. ${ }^{23}$ retrospectively analyzed 81 critically ill patients with COVID-19 pneumonia in the ICU and found an incidence of $25 \%$ of VTE. Klok et al. ${ }^{24}$, found an incidence of $31 \%$ of thrombotic complications in ICU patients with COVID-19 infections despite thrombosis prophylaxis, this percentage being remarkably high. Middeldorp et al. ${ }^{25}$ stated that the risk of VTE in patients with COVID-19 is high, especially in patients hospitalized in the ICU, and that adopting a screening strategy with diagnostic tests at a low threshold may be a good approach. It is thus clear that a joint effort between local professionals working in the 
field of Angiology and Vascular Surgery is necessary so that a collaborative network can increasingly improve, at the collective level, the carried out procedures and care flowcharts, in addition to assisting in the remodeling of services provided during the COVID-19 pandemic.

\section{CONCLUSION}

COVID-19 can predispose to venous and arterial thromboembolic diseases due to excessive inflammation, hypoxia, immobilization and DIC. Therefore, there is a need for risk stratification for VTE for all hospitalized patients through the use of scores such as the Caprinis, in addition to the complementary use of scores to stratify the risk of bleeding.

Note that thromboprophylaxis is necessary during the hospitalization of patients with COVID-19, unless there is a contraindication, the choice and duration of therapy varying according to the patient's clinical situation and the protocol established in the hospital unit, and based on the guidelines of the Hemostasis and Thrombosis Societies. The post-discharge thromboprophylaxis of the patient with COVID-19 remains lacking in data, with no consensus, and the decision for its use and DD cutoff point that guides its choice must be made individually, always analyzing the risk of thrombotic or bleeding events, according to established institutional protocols based on previous safe evidence.

In addition to the clinical management of vascular and hematological complications that SarsCoV-2 infection can cause, vascular surgery departments need to readjust their routines by creating or reviewing algorithms and protocols that make their performance possible, even if restricted, during the current world outbreak of COVID-19.

\section{R E S U M O}

A epidemia pelo novo Coronavirus (2019-nCoV), surgido na cidade de Wuhan, na China, em dezembro de 2019, quando sintomática, apresenta-se majoritariamente por um quadro de pneumonia pulmonar que é precedida por febre, tosse seca e mialgia. No entanto, conforme a doença se espalhou globalmente e o número de hospitalizações aumentaram de forma exponencial, notou-se que a maior parte dos pacientes graves internados por COVID-19 possuem alterações laboratoriais dignas de atenção, como linfopenia, neutrofilia, aumento do tempo de protrombina e elevação dos níveis de D-dímero. Devido tais mudanças se mostrarem cruciais para a taxa de mortalidade e morbidade nesse subgrupo de infectados, diversos trabalhos com enfoque na fisiopatologia, principalmente hematológica, da doença surgem a cada dia. Aprofundando em tais estudos, variados trabalhos publicados evidenciaram a infecção pelo Sars-CoV-2 à instalação de um estado pró-trombótico em pacientes hospitalizados graves, o que acarreta em potencial ocorrência de eventos trombóticos venosos ou arteriais nessa coorte. Assim, para entender como os Departamentos de Angiologia e Cirurgia Vascular estão atuando no contexto da pandemia de COVID-19, este estudo tem por objetivo reunir estudos que revelam desde protocolos aplicados nos serviços vasculares na atual conjuntura, até a atuação dos cirurgiões vasculares e angiologistas no manejo clínico e cirúrgico de pacientes infectados ou não, como forma de ajudar e esclarecer essa especialidade durante o contexto de pandemia pelo novo coronavírus. Para a seleção dos trabalhos foram utilizados os seguintes critérios de busca: "Coronavirus and venous thrombosis", "Coronavirus and thrombosis", "COVID-19 and venous thrombosis" e "COVID-19 Coronavirus and thrombosis".

Palavras chave: Infecções por Coronavirus. Coagulação Sanguínea. Trombose. Embolia Pulmonar. Coronavirus.

\section{REFERENCES}

1. World Health Organization. WHO Coronavirus diseases (COVID-19) Dashboard [Internet]. 2020 [acesso em 28 abr 2020]. Disponível em: https:// covid19.who.int/.

2. Huang C, Wang Y, Li X, Ren L, Zhao J, Hu Y, et al. Clinical features of patients infected with 2019 novel coronavirus in Wuhan, China. Lancet. 2020;
395(10223):497-506.

3. Lippi G, Plebani M. Laboratory abnormalities in patients with COVID-2019 infection. Clin Chem Lab Med. 2020 Mar 3. doi: 10.1515/cclm-2020-0198.

4. Zhou F, Yu T, Du R, Fan G, Liu Y, Liu Z, et al. Clinical course and risk factors for mortality of adult inpatients with COVID-19 in Wuhan, China: a retrospective cohort study. Lancet. 2020;395(10229):1054-62.

5. Han $H$, Yang L, Liu R, Liu F, Wu K, Li J, et al. 
Prominent changes in blood coagulation of patients with SARS-CoV-2 infection. Clin Chem Lab Med. 2020 Mar 16. doi: 10.1515/cclm-2020-0188.

6. Tang N, Li D, Wang X, Sun Z. Abnormal coagulation parameters are associated with poor prognosis in patients with novel coronavirus pneumonia. J Thromb Haemost. 2020;18(4):844-7.

7. Obi AT, Barnes GD, Wakefield TW, Brown Rvt S, Eliason JL, Arndt E, et al. Practical diagnosis and treatment of suspected venous thromboembolism during COVID-19 Pandemic. J Vasc Surg Venous Lymphat Disord. 2020 Apr 16. doi: 10.1016/j. jvsv.2020.04.009.

8. Pannucci CJ, Swistun L, MacDonald JK, Henke PK, Brooke BS. Individualized venous thromboembolism risk stratification using the 2005 Caprini score to identify the benefits and harms of chemoprophylaxis in surgical patients: a meta-analysis. Ann Surg. 2017;265(6):1094-103.

9. Casini A, Alberio L, Angelillo-Scherrer A, Fontana P, Gerber B, Graf L, et al. Thromboprophylaxis and laboratory monitoring for in-hospital patients with COVID-19 - A Swiss Consensus Statement by the Working Party Hemostasis. Swiss Med Wkly. 2020 Apr 11;150:w20247. doi:10.4414/ smw.2020.20247.

10. Marietta M, Ageno W, Artoni A, De Candia E, Gresele P, Marchetti $M$, et al. COVID-19 and haemostasis: a position paper from Italian Society on Thrombosis and Haemostasis (SISET). Blood Transfus. 2020 Apr 8. doi: 10.2450/2020.0083-20.

11. Wu Z, McGoogan JM. Characteristics of and important lessons from the coronavirus disease 2019 (COVID-19) outbreak in China: summary of a report of 72,314 cases from the Chinese Center for Disease Control and Prevention. JAMA. 2020;323(13):1239-1242. doi: 10.1001/ jama.2020.2648.

12. Thachil J, Tang N, Gando S, Falanga A, Cattaneo M, Levi $M$, et al. ISTH interim guidance on recognition and management of coagulopathy in COVID-19. J Thromb Hemost. 2020;18(5):1023-6.

13. Testa S, Paoletti O, Giorgi-Pierffranceschi M, Pan A. Switch from oral anticoagulants to parenteral heparin in SARS-COV-2 hospitalized patients. Intern
Emerg Med. 2020 Apr 15. doi: 10.1007/s11739020-02331-1.

14. Tan GWL, Chandrasekar S, Lo ZJ, Hong Q, Yong E, Lingam $P$, et al. Early Experience in the COVID-19 pandemic from a Vascular Surgery Unit in a Singapore Tertiary Hospital. J Vasc Surg. 2020 Apr 17. doi: 10.1016/j.jvs.2020.04.014.

15. American College of Surgeons [Internet]. Chicago (IL): ACS; c1996-2020. COVID-19: Recommendations for Management of Elective 17 Surgical Procedures [about 2 p.]. Available from: https://www.facs.org/about-acs/covid19/ information-for-surgeons/elective-surgery

16. Mirza AK. Perspectives on vascular surgical practice change due to COVID-19 at a nonacademic tertiary care center. J Vasc Surg. 2020 Apr 17: S07415214(20)30592-9. doi: 10.1016/j.jvs.2020.04.016.

17. Chisci E, Masciello F, Michelagnoli S. Creation of a vascular surgical Hub responding to the COVID-19 emergency: the Italian USL Toscana Centro model. J Vasc Surg. 2020 Apr 16:S0741-5214(20)30605-4. doi: 10.1016/j.jvs.2020.04.019.

18. Wang C, Horby PW, Hayden FG, Gao GF. A novel coronavirus outbreak of global health concern. Lancet. 2020;395(10223):470-3.

19. Zhou W, Liu YS, Tian D, Wang C, Cheng J, Hu M, et al. Potential benefits of precise corticosteroids therapy for severe 2019-nCoV pneumonia. Signal Transduct Target Ther. 2020;5(1):18. doi: 10.1038/ s41392-020-0127-9

20. Wan S, Yi Q, Fan S, Lv J, Zhang X, Guo L, et al. Characteristics of lymphocyte subsets and cytokines in peripheral blood of 123 hospitalized patients with 2019 novel coronavirus pneumonia (NCP). MedRxiv. 2020 Feb 12. doi: 10.1101/2020.02.10.20021832.

21. Shi $C$, Wang $C$, Wang $H$, Yang $C$, Cai F, Zeng F, et al. The potential of low molecular weight heparin to mitigate cytokine storm in severe COVID-19 patients: a retrospective clinical study. Medrxiv. 2020 Apr 15. doi: 10.1101/2020.03.28.20046144v3.

22. Magro C, Mulvey JJ, Berlin D, Nuovo G, Salvatore S, Harp J, et al. Complement associated microvascular injury and thrombosis in the pathogenesis of severe COVID-19 infection: a report of five cases. Transl Res. 2020 Apr 15; S1931-5244(20)30070-0. doi: 
10.1016/j.trsl.2020.04.007.

23. Cui S, Chen S, Li X, Liu S, Wang F. Prevalence of venous thromboembolism in patients with severe novel coronavirus pneumonia. J Thromb Haemost . 2020 Apr 9. doi: 10.1111/jth.14830.

24. Klok FA, Kruip MJHA, van der Meer NJM, Arbous MS, Gommers DAMPJ, Kant KM, et al. Incidence of thrombotic complications in critically ill ICU

Received in: 15/04/2020

Accepted for publication: 28/04/2020

Conflict of interest: no.

Funding source: none. patients with COVID-19. Thromb Res. $2020 \mathrm{Apr}$ 10;S0049-3848(20)30120-1. doi: 10.1016/j. thromres.2020.04.013

25. Middeldorp S, Coppens $M$, van Haaps TF, Foppen M, Vlaar AP, Müller MC, et al. Incidence of venous thromboembolism in hospitalized patients with COVID-19. J Thromb Haemost. 2020 May 5. doi: 10.1111/jth.14888. Online ahead of print.

\section{Mailing address:}

Bernardo Cunha Senra Barros

E-mail: drbernardobarros@gmail.com 